\title{
厄尔尼诺-南方涛动研究新进展
}

陈大可 ${ }^{1,2,3^{*}}$, 连涛 1,3

1. 自然资源部第二海洋研究所, 卫星海洋环境动力学国家重点实验室, 杭州 310012;

2. 南方海洋科学与工程广东省实验室(珠海), 珠海 519000;

3. 上海交通大学海洋学院, 上海 200240

* 联系人, E-mail: dchen@ sio.org.cn

\section{Frontier of El Niño-Southern Oscillation research}

\author{
Dake Chen $^{1,2,3^{*}} \&$ Tao Lian ${ }^{1,3}$ \\ ${ }^{1}$ State Key Laboratory of Satellite Ocean Environment Dynamics, Second Institute of Oceanography, Ministry of Natural Resources, Hangzhou \\ 310012, China; \\ ${ }^{2}$ Southern Marine Science and Engineering Guangdong Laboratory (Zhuhai), Zhuhai 519000, China; \\ ${ }^{3}$ School of Oceanography, Shanghai Jiao Tong University, Shanghai 200240, China \\ * Corresponding author, E-mail: dchen@ sio.org.cn \\ doi: 10.1360/TB-2020-1219
}

厄尔尼诺-南方涛动(ENSO)是地球气候系统中最强烈 的年际振荡, 对包括我国在内的全球大部分地区的天气及 气候有举足轻重的影响, 因而长期以来一直是海洋与大气 科学研究的焦点. 在National Science Review 《《国家科学评 论》) 2018 年第 5 卷第 6 期的 ENSO专题上, 多位科学家对 ENSO的理论、预测、影响等方面的研究进展做了全方位 的综述 ${ }^{[1 \sim 3]}$, 并探讨了ENSO在古气候和全球变暖背景下的 变化、ENSO与热带气旋的相互作用、ENSO观测系统的建 设与改进等前沿科技问题 ${ }^{[47]}$. ENSO研究的先驱 Mark Cane教授也在专题中分享了他的科学洞见和不同寻常的 科研经历. 该专题发表以来, 受到国内外学术界的广泛关 注, 期间又涌现出一批颇具挑战性的研究成果. 我们对这 些最新成果作一个简略的梳理, 重点介绍ENSO动力学、副 热带太平洋对ENSO的影响、基于机器学习的ENSO预测等 研究方向的重要进展, 并对今后应该关注的问题提出一些 看法.

在经典ENSO理论中, 其演变受控于热带太平洋的海 气正反馈过程 ${ }^{[8]}$ 以及上层海洋热含量的变化 ${ }^{[9,10]}$. 其中热 含量的变化通常超前一年, 因此被认为是预测ENSO的重 要前期信号. $\mathrm{Hu}$ 等人 ${ }^{[11]}$ 最近指出, 在1979、2004、2014及 2018年厄尔尼诺期间, 热带太平洋大气环流罕见地没有因 为厄尔尼诺的发生而表现出大范围异常, 特别是赤道太平 洋对流区没有东移到赤道日界线附近. 这是因为这些事件 发生时赤道太平洋纬向海表温度(SST)异常梯度较小, 从 而难以激发大尺度大气环流. 考虑到此类非耦合型厄尔尼
诺对气候系统的影响与传统的耦合型厄尔尼诺截然不同, 在评价模式模拟和预测水平时除了几个传统的ENSO指标 外，也应考虑纬向SST异常指标. 在ENSO期间热带太平洋 上层海洋热含量变化的问题上, Neske和 McGregor ${ }^{[12]}$ 通过 海洋模式试验发现, 西边界反射和即时风场对热含量变化 的贡献相当，这说明仅凭年初热含量的信息不足以准确预 测当年ENSO的强弱. Larson和Pegion ${ }^{[13]}$ 利用耦合模式集合 试验进一步指出, 无论年初热含量是增加还是减少, 模式 预测的ENSO都有非常大的不确定性. 因此, 海洋的初始 热状态对于ENSO预测也许不像经典理论预示得那么重要.

关于副热带太平洋对ENSO的影响, Amaya等人 ${ }^{[14]}$ 发 现北太平洋SST异常信号可以通过风-蒸发反馈 ${ }^{[15]}$ 和Gill型 响应 ${ }^{[16]}$ 影响赤道海表风场, 进而影响ENSO演变. Chakravorty等人 ${ }^{[17]}$ 也指出, 在北太平洋振荡的正相位期间, 其所 伴随的副热带西风异常会通过减弱信风的方式促进厄尔 尼诺产生. Ding等人 ${ }^{[18]}$ 发现, 当考虑北太平洋和南太平洋 的内在模态时, 能改进ENSO预测效果. Hou等人 ${ }^{[19]}$ 则利用 多个耦合模式的资料从可预报性最优误差增长的角度, 验 证了北太平洋和南太平洋模态对ENSO预测的重要性. Hua 和 $\mathrm{Su}^{[20]}$ 指出模式在东南太平洋的初始冷误差会通过局地 海气相互作用放大, 从而造成预测失误. 此外, 西太平洋 热带气旋与ENSO之间的跨尺度相互作用也是近年来的研 究热点. Wang等人 ${ }^{[21]}$ 通过数据分析发现西太平洋热带气 旋能增强厄尔尼诺强度. Lian等人 ${ }^{[22]}$ 指出, 有超过 $50 \%$ 的 西太平洋热带气旋能通过增强赤道西风异常的方式将局 
地信风反转; 当把热带气旋风场加人到耦合模式中后, 模 拟的ENSO呈现出明显的不规则性及非对称性(图1). 尽管 模式中海洋热含量的 “充-放”依然与ENSO指数高度相关, 但每一次ENSO事件的强度甚至冷暖位相都明显受热带气 旋个数与发生时刻的影响. 这些结果证实西太平洋热带气 旋与ENSO之间存在强相互作用, 因而ENSO预测系统应当 考虑西太平洋热带气旋的跨尺度作用.

这些研究结果对现有的基于热带太平洋海气正反馈 及上层海洋热含量变化而构建的ENSO理论及预测模式提 出了严峻挑战. 那么, 是否可以避免理论的塩筴, 发展完 全基于数据但不同于传统统计模式的预测方法? 随着近年 来迅速发展的大数据及机器学习方法, 一些学者开始尝试 利用机器学习提高ENSO预测技巧. Ham 等人 ${ }^{[23]}$ 基于再分 析和模式资料中的全球SST和海洋热含量数据, 通过卷积 神经网络方法训练模式, 获得了比目前主流气候模式更高 的ENSO预测技巧, NINO3.4指数提前 12 个月预测的相关系 数超过 0.65. Petersik和Dijkstra ${ }^{[24]}$ 利用神经网络方法训练小 数据量的ENSO指数和海洋热含量, 也得到了类似的预测 能力. 最新一项工作则是结合经验模态分解与时序卷积网 络方法, 进一步将NINO3.4指数提前 12 个月的相关系数提 高到 $0.83^{[25]}$. 尽管基于机器学习的ENSO预测模型看似达 到了相当可观的水平, 但其本质还是数据驱动的统计训
练, 很大程度上依赖于数据样本的大小. 问题是实际观测 的ENSO样本太少，只能依靠替代资料和动力耦合模式的 输出结果来进行模式训练. 这类基于机器学习的模型虽然 可以节省计算资源, 但其预测能力原则上很难突破动力模 式的上限. 此外, 机器学习难以从动力和机制上揭示预报 误差的来源.

上述研究进展表明, ENSO研究领域仍然充满活力, 仍有很多悬而未决的问题需要深人探索. 我们认为, 今后 的研究应利用最新的观测和再分析数据, 结合模式试验, 进一步深化对ENSO动力机制和可预测性的理解. 具体来 说, 至少应该在以下几个方面发力: 第一, 定量分析热带 太平洋海气正反馈、上层海洋热含量这两个在传统意义上 对ENSO演变最为重要的过程; 第二, 揭示副热带太平洋 以及其他大洋通过大气桥和海洋通道对ENSO的影响, 并 将这些影响从理论上纳人ENSO动力框架之中; 第三, 阐 明海气耦合气候模式中系统性偏差的成因, 并通过动力过 程矫正和资料同化 ${ }^{[26,27]}$ 消除这些偏差来提高模式的可靠 性和预测能力; 第四, 将一些近年来发现的对ENSO有重 要影响的跨尺度海气耦合过程, 比如热带气旋与ENSO的 相互作用, 纳人到机器学习的训练集合中, 一方面增加基 础数据的自由度, 另一方面将机器学习和物理认知相结 合, 从而构建更加科学的人工智能预测模型.

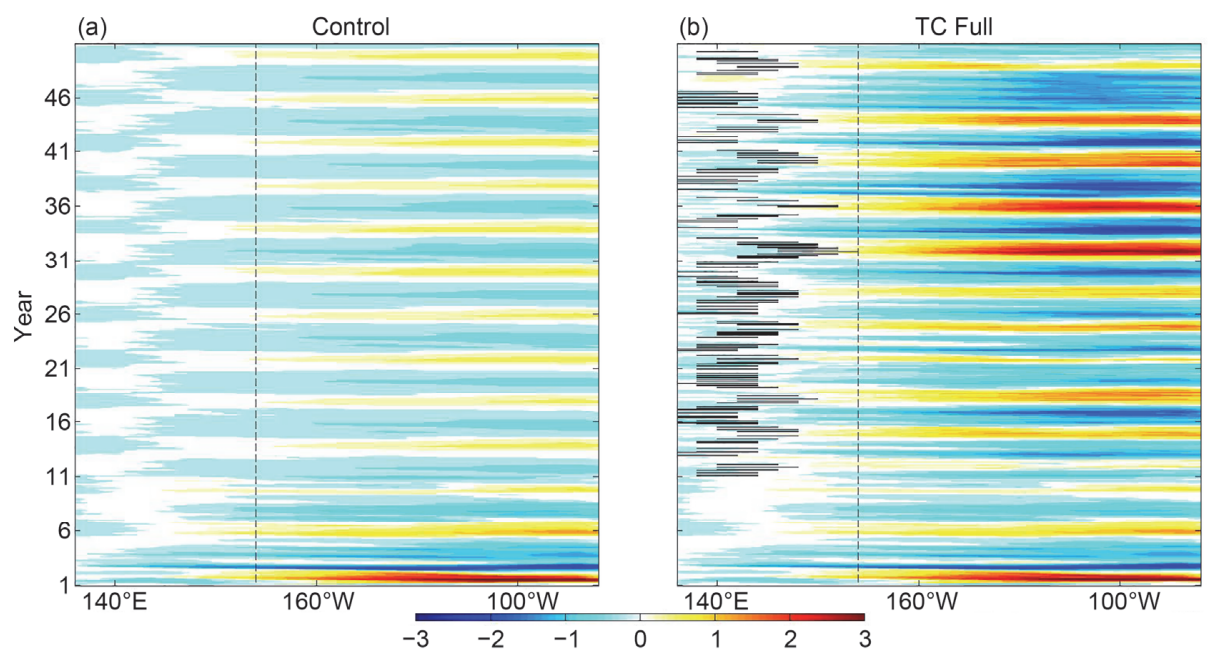

图 1 海气耦合模式中赤道区域 $\left(5^{\circ} \mathrm{S} 5^{\circ} \mathrm{N}\right)$ 平均海表温度异常的演变. (a) 控制试验; (b) 热带气旋试验. 热带气旋从第 11 年开始加人, 黑实线 是气旋在赤道上的投影. 热带气旋对ENSO强度、不规则性和非对称性的影响显而易见

Figure 1 Evolution of equatorial $\left(5^{\circ} \mathrm{S}-5^{\circ} \mathrm{N}\right)$ SST anomalies in the Control (a) and TC Full (b) runs. The TCs are added from model year 11. Black lines in (b) denote TC-related wind stresses. The influence of TCs on ENSO magnitude, irregularity, and asymmetry is obviously seen

\section{参考文献}

1 Wang C. A review of ENSO theories. Natl Sci Rev, 2018, 5: 813-825 
2 Tang Y, Zhang R, Liu T, et al. Progress in ENSO prediction and predictability study. Natl Sci Rev, 2018, 5: 826-839

3 Yang S, Li Z, Yu J, et al. El Niño-Southern Oscillation and its impact in the changing climate. Natl Sci Rev, 2018, 5: 840-857

4 Lu Z, Liu Z. Examining El Niño in the Holocene: Implications and challenges. Natl Sci Rev, 2018, 5: 807-809

5 Lian T, Chen D, Ying J, et al. Tropical Pacific trends under global warming: El Niño-like or La Niña-like? Natl Sci Rev, 2018, 5: 810-812

6 Chen D, Smith N, Kessler W. The evolving ENSO observing system. Natl Sci Rev, 2018, 5: 805-807

7 Chen D, Lian T. Interaction of western Pacific tropical cyclone with El Niño diversity. Natl Sci Rev, 2018, 5: 803-804

8 Bjerknes J. Atmospheric teleconnections from the equatorial Pacific. Mon Weather Rev, 1969, 97: 163-172

9 Wyrtki K. El Niño-The dynamic response of the equatorial Pacific Ocean to atmospheric forcing. J Phys Oceanogr, 1975, 5: 572-594

10 Jin F. An equatorial ocean recharge paradigm for ENSO. Part I: Conceptual model. J Atmos Sci, 1997, 54: 811-829

11 Hu Z, McPhaden M J, Kumar A, et al. Uncoupled El Niño warming. Geophys Res Lett, 2020, 47: e2020GL087621

12 Neske S, McGregor S. Understanding the warm water volume precursor of ENSO events and its interdecadal variation. Geophys Res Lett, 2018, 45: 1577-1585

13 Larson S M, Pegion K. Do asymmetries in ENSO predictability arise from different recharged states? Clim Dyn, 2020, 54: 1507-1522

14 Amaya D J, Kosaka Y, Zhou W, et al. The North Pacific pacemaker effect on historical ENSO and its mechanisms. J Clim, 2019, 32: 7643-7661

15 Xie S P, Philander S. A coupled ocean-atmosphere model of relevance to the ITCZ in the eastern Pacific. Tellus, 1994, 46: 340-350

16 Gill A E. Some simple solutions for heat-induced tropical circulation. Quart J Roy Meteorol Soc, 1980, 106: 447-462

17 Chakravorty S, Perez R, Anderson B, et al. Testing the trade wind charging mechanism and its influence on ENSO variability. J Clim, 2020, 33: 7391-7411

18 Ding R, Tseng Y, Li J, et al. Relative contributions of North and South Pacific sea surface temperature anomalies to ENSO. J Geophys Res Atmos, 2019, 124: 6222-6237

19 Hou M, Duan W, Zhi X. Season-dependent predictability barrier for two types of El Niño-Southern Oscillation events revealed by an approach to data analysis for predictability. Clim Dyn, 2019, 53: 5561-5581

20 Hua L, Su J. Southeastern Pacific error leads to failed El Niño forecasts. Geophys Res Lett, 2020, 47: e2020GL088764

21 Wang Q, Li J, Jin F, et al. Tropical cyclones act to intensify El Niño. Nat Commun, 2019, 10: 3793

22 Lian T, Ying J, Ren H, et al. Effects of tropical cyclones on ENSO. J Clim, 2019, 32: 6423-6443

23 Ham Y, Kim J, Luo J. Deep learning for multi-year ENSO forecasts. Nature, 2019, 573: 568-572

24 Petersik P, Dijkstra H. Probabilistic forecasting of El Niño using neural network models. Geophys Res Lett, 2020, 47: e2019GL086423

25 Yan J, Mu L, Wang L, et al. Temporal convolutional networks for the advance prediction of ENSO. Sci Rep, 2020, 10: 8055

26 Tao L, Duan W. Using a nonlinear forcing singular vector approach to reduce model error effects in ENSO forecasting. Weather Forecast, 2019, 34: 1321-1342

27 Tao L, Duan W, Vannitsem S. Improving forecasts of El Niño diversity: A nonlinear forcing singular vector approach. Clim Dyn, 2020, 55: 739-754 